balance impairment and cognitive impairment at admission had negligible effects on resident disablement over two years.

Conclusions: Residents who are more disabled at admission experience slower disablement over two years than residents who are less disabled at admission. This rate difference may reflect an untapped opportunity for slowing disablement among residents who are admitted to long-term care with lower disability.

\section{FACTORS ASSOCIATED WITH ANTIPSYCHOTIC MEDICATION USE IN LONG-STAY NURSING HOME RESIDENTS}

L.J. Phillips, G.F. Petroski, M. Rantz, N.M. Birtley, C. Siem, University of Missouri, Columbia, Missouri

Nationally, $17 \%$ of nursing home residents without qualifying diagnoses of schizophrenia, Huntington's' Disease, and/or Tourette's Syndrome receive antipsychotic medications. Identifying factors associated with antipsychotic use could reveal targets for alternative interventions. Using the Missouri Minimum Data Set collected between 1/1/2015 and $12 / 31 / 2015$, variables correlated with antipsychotic use in long-stay nursing home residents age $\geq 65$ years, lacking a qualifying diagnosis, and not comatose, were examined. Among 36,307 residents meeting inclusion criteria, 18\% received an antipsychotic. After dividing the sample into 10 random equivalently-sized subsets, logistic regression with forward selection of candidate variables associated $(\mathrm{p}<0.01)$ with antipsychotic use was run for each data subset. Variables selected in $\geq 50 \%$ of subsets were included in a final logistic regression model using 36,307 cases. Statistically significant predictors of antipsychotic use, in ascending order of absolute magnitude ranging from odds ratios of 0.96 to 6.22, were fewer late-loss activities of daily living, lower age, non-Alzheimer's dementia, antidepressant medication, cognitive impairment, wandering, antianxiety medication, behavioral symptoms, psychosis symptoms, psychotic disorder, and manic depression. The area under the receiver operator characteristic curve was 0.81 , indicating good discrimination between residents receiving and not receiving antipsychotics. This study identified characteristics of residents most at risk for antipsychotic use; these findings could guide nursing homes in proactively planning need-based interventions. By implementing interventions targeted toward younger, higherfunctioning dementia residents, and including relaxation and cognitive behavioral therapy techniques for anxiety and depression, antipsychotic use may be reduced, thereby mitigating harm caused by medication side effects and complying with federal regulations.

\section{PATTERNS OF NEW PHYSICAL PROBLEMS EMERGING IN LONG-TERM CARE RESIDENTS WITH DEMENTIA}

C. Kovach ${ }^{1,2}$, J. Ellis ${ }^{1}$, C. Venes ${ }^{1}, 1$. Nursing, University of Wisconsin-Milwaukee, Milwankee, Wisconsin, 2. Jewish

Home and Care Center, Milwaukee, Wisconsin

People receiving skilled nursing care have multiple comorbid conditions that impact comfort and resource utilization. This longitudinal study describes variations in the trajectories of new physical problems emerging over 8 weeks and the predictive value for future health and behavior in residents from two nursing homes with dementia. The sample of
72 had 668 new physical problems with a range of 2 to 37 occurring over eight weeks. Sixty-five percent of the sample $(\mathrm{n}=47)$ had 5 or more new problems and were identified by three unstable trajectories. Thirty-nine percent $(n=28)$ had a spike in new problems of 4 or more in one week. Spikes, new physical problems, and the problem-free duration predicted $23.6 \%$ of the variance in both new physical problems and new agitation occurring in the subsequent month $(\mathrm{p}<.001)$. Common problems, acute illness, and symptoms accounted for $28.2 \%$ of the variance in subsequent new physical problems $(\mathrm{p}<.001)$ and $25.7 \%$ of the variance in subsequent agitation $(\mathrm{p}<.001)$. The frequency of new problems and differences in the patterns of how these problems emerged over a relatively short time period suggests instability and variability in physiologic health that has not been previously identified. The possible existence of different pathways of decline may have important implications for health care delivery. Findings suggest a higher intensity of need for skilled assessment and treatment than may be available in many long-term care organizations.

\section{THE EFFECT OF ENVIRONMENTAL ADAPTATIONS ON SELF-REPORTED BATHING DISABILITY AND INSTITUTIONALIZATION}

W.K. Graham, M. Bilger, Duke-NUS Medical School, Singapore, Singapore

Disability in bathing, an activity of daily living, has been shown to be an independent predictor of long-term nursing home (LTNH) admission. It is theorized that environmental adaptations (EA) in the home can reduce the physical demands of bathing and hence delay the development of this disability. This study aims to examine the effect of home environmental adaptations (EA) on bathing disability and subsequently on the probability of LTNH admission among older Americans with bathing disability.

This study uses data from Waves 8 to 11 (years 20062012) of the Health and Retirement Study (HRS). The analysis, based on responses to the experimental survey module on Characteristics of the Home Environment $(\mathrm{N}=1616)$, is conducted in the framework of logistic models explaining selfreported disabilities as functions of related impairments and EAs. The mitigating effect of EAs on self-reported disabilities is measured via interaction terms between impairments and EAs and their significance tested using the $t$ statistic.

Findings show that among people who have difficulty walking across a room, the presence of grab bars in the shower or tub area and of separate shower stalls is associated with lower probability of self-reported bathing disability. Among people who have difficulty stooping, kneeling and crouching, those who have separate shower stalls are also less likely to report having bathing disability. These results suggest that, by reducing disability rates, grab bars in the shower or tub area and separate shower stalls can facilitate aging-in-place and lower LTNH admission rates.

\section{A COMMUNICATION INTERVENTION TO REDUCE RESISTIVENESS IN DEMENTIA CARE}

K.N. Williams ${ }^{2}$, Y. Perhounkova ${ }^{3}$, R. Herman ${ }^{2}$, A.L. Bossen ${ }^{3}$, 2. The University of Kansas Medical Center, Kansas City, Kansas, 2. University of Kansas, Lawrence, Kansas, 3. The University of Iowa, Iowa City, Iowa 